\title{
Analyses physiques et patrimoine culturel
}

\author{
Ina Reiche \\ Laboratoire du Centre de Recherche et de Restauration des Musées de France \\ Palais du Louvre, 75001 Paris
}

\begin{abstract}
Matériaux complexes et souvent hétérogènes, les objets d'art ou archéologiques constituent un défi pour l'analyse physico-chimique. Dans cet article, quelques exemples sont présentés, illustrant la nécessité de stratégies en différentes étapes combinant des méthodes complémentaires. Dans de nombreux cas, il est nécessaire de recourir à des techniques de microanalyse (microscopie électronique à balayage, microfluorescence $X, . .$.$) et parfois même d'utiliser les$ grands instruments, comme les accélérateurs de particules ou le rayonnement synchrotron.
\end{abstract}

\section{Introduction}

Les objets du patrimoine étudiés en sciences humaines donnent un grand nombre d'informations sur notre passé grâce à l'analyse de leur style, à la connaissance de leur contexte historique et archéologique et à la comparaison avec les sources écrites anciennes. Par ailleurs, des renseignements précieux sur leur origine, leur mode de fabrication et leur âge peuvent être cachés dans la composition chimique et la structure à diverses échelles des matériaux qui les constituent. Ainsi, une nouvelle démarche, la " science du patrimoine » ou « archéométrie », utilisant plusieurs techniques expérimentales complémentaires, a été développée avec pour objectifs :

- de déterminer la provenance et l'âge des objets, ainsi que la nature exacte des matériaux impliqués,

- de révéler les techniques de fabrication de ces objets et matériaux,

- et d'étudier les phénomènes d'altération, afin de mieux évaluer la qualité des informations déduites des œuvres et de définir les facteurs majeurs permettant leur conservation.

Ces recherches comprennent aussi l'adaptation de nouvelles méthodes analytiques aux spécificités liées aux œuvres d'art et aux objets archéologiques. En particulier :

- les matériaux (métaux, pierres, céramiques, verres, ossements, colorants, pigments,...) constituant ces œuvres ou objets sont généralement composites et hétérogènes et ont été transformés par l'homme ou altérés au cours du temps ;

- les critères permettant de déterminer l'origine d'un matériau ou d'identifier un procédé de fabrication particulier, sont souvent des éléments présents à l'état de traces, des microinclusions ou parfois même des morphologies particulières de nanocristallites ;

- enfin, le caractère précieux de certaines œuvres interdit alors d'effectuer des prélèvements.

La méthodologie analytique à adopter sera donc définie selon le problème posé, le type d'objet ou le matériau à étudier, la possibilité d'effectuer ou non un prélèvement, la quantité de matière disponible, et la complexité et le degré de dégradation du matériau. Généralement, il sera nécessaire de recourir à diverses techniques complémentaires, d'effectuer des examens à différentes échelles, et d'établir des bases de données à partir de matériaux anciens connus [1].

Nous présentons ici brièvement les méthodes d'examen et d'analyse couramment employées en archéométrie et quelques exemples de recherche, illustrant l'approche utilisée et montrant l'importance des méthodes physiques dans la résolution de questions et mystères en art et en archéologie.

\section{Méthodes d'investigation}

Le tableau 1 donne un aperçu des méthodes d'examen microscopique et d'analyse les plus couramment utilisées dans ces recherches. Les méthodes de datation et les analyses biomoléculaires (ADN ancienne par exemple) sont omises, de nombreux ouvrages et articles décrivant en détail leurs applications en archéologie et histoire de l'art [2-4].

Tout d'abord, l'observation de la surface des tableaux ou objets sous lumière directe ou rasante, sous rayonnement ultraviolet (UV) et infrarouge (IR), ainsi qu'en volume par radiographie $X$, donne des renseignements très précieux sur l'état de conservation général, les fissures éventuelles, les parties qui se détachent de la surface, les brunissements, le craquelé, les zones restaurées, les "repentirs ", les dessins sousjacents et également le support [5]. Puis, les méthodes de microscopie optique ou électronique (à balayage ou en transmission) permettent d'observer plus en détail (à l'échelle du micromètre ou même du nanomètre) des zones sélectionnées. Enfin, dans le cas des matériaux inorganiques, les analyses élémentaires ${ }^{1}$, structurales et spectroscopiques, réalisées sur des microprélèvements ou directement sur des zones choisies dans les objets, donnent accès respectivement à la composition chimique, à l'arrangement atomique et à la structure électronique (état de valence, par exemple) des espèces chimiques contenues dans les différentes phases constituant le matériau. Parmi ces méthodes, les techniques moins classiques d'analyse par faisceau d'ions (IBA) [6], ou encore celles utilisant des sources synchrotron, sont de plus en plus employées, car elles sont très sensibles aux éléments traces $[7,8]$. Quant aux matériaux organiques, les méthodes d'analyse moléculaire, telle que la chromatographie en phase gazeuse couplée à la spectrométrie de masse, permettent d'identifier les marqueurs des composés organiques de départ, et ceux dus à une transformation par l'homme ou à une dégradation [9].

\section{De la microfluorescence $X$ (MFX) résolue en deux dimensions à la MFX 3D}

\section{Le dessin à la pointe de métal dans les ateliers des grands maîtres}

À l'époque du haut Moyen-Âge et du début de la Renaissance, deux matériaux étaient principalement connus pour le dessin : l'encre et la pierre noire. Un troisième matériau, moins courant et plus inattendu, fut cependant également employé : la pointe de métal (utilisée ici non pas comme un outil de gravure, mais comme un outil de dessin, analogue par exemple à un crayon à mine de plomb). Son apogée se situe au $X V^{e}$ et dans la première moitié du $X V I^{e}$ siècles, à la suite desquels cet instrument de dessin a été remplacé par d'autres matériaux moins contraignants.

Les dessins à la pointe de métal n'avaient jamais fait l'objet d'investigations analytiques approfondies. De nombreuses interrogations subsistaient donc : quels métaux et alliages ont été utilisés? Une préparation des supports (parchemin puis

1 Analyses permettant la détermination de la composition chimique des matériaux d'une manière qualitative et quantitative. 
papier) était-elle nécessaire pour les rendre abrasifs ? Existaitil des pratiques ou des matériaux caractéristiques de certains artistes ou de certaines écoles ? Dans le but d'apporter des éléments de réponse à ces questions, plus d'une centaine d'œuvres conservées dans différents musées français et allemands ont été analysées [10-11].

L'analyse des matériaux graphiques est soumise à des contraintes particulières : elle doit être totalement non-des- tructive, ce qui implique une analyse directe de l'œuvre sans prélèvement et sans dommages induits. Elle doit, de plus, caractériser une très faible quantité de matière (quelques dizaines de $\mu \mathrm{g}$ ) déposée sur un support (figure 1) qui, luimême, peut contenir des éléments chimiques communs à ceux des tracés. II s'agit donc d'un véritable défi analytique.

Une technique d'analyse adaptée à l'étude des dessins à la pointe de métal est la spectrométrie de fluorescence $\mathrm{X}$ indui-

\begin{tabular}{|c|c|c|c|c|c|c|}
\hline $\begin{array}{l}\text { Technique } \\
\text { d'examen et } \\
\text { d'analyse }\end{array}$ & $\begin{array}{l}\text { Type de } \\
\text { méthode }\end{array}$ & $\begin{array}{l}\text { Prélèvement } \\
\text { nécesssaire }\end{array}$ & Zone d'analyse & $\begin{array}{c}\text { Meilleure } \\
\text { résolution spatiale } \\
\text { de l'analyse }\end{array}$ & $\begin{array}{l}\text { Meilleure limite } \\
\text { de détection }\end{array}$ & $\begin{array}{c}\text { Apparei } \\
\text { portable } \\
\text { (analyse } \\
\text { in situ) }\end{array}$ \\
\hline $\begin{array}{l}\text { Microscope optique } \\
\text { (MO) }\end{array}$ & $\begin{array}{c}\text { Examen } \\
\text { microscopique }\end{array}$ & oui & surface & qq $10 \mu m^{2}$ & - & non \\
\hline $\begin{array}{c}\text { Microscopie } \\
\text { électronique à } \\
\text { balayage (MEB) } \\
\text { couplée à analyse X } \\
\text { dispersive en } \\
\text { énergie (EDX) }\end{array}$ & $\begin{array}{c}\text { Examen } \\
\text { microscopique } \\
\text { et analyse } \\
\text { élémentaire } \\
\text { locale }\end{array}$ & $\begin{array}{c}\text { oui (coupe } \\
\text { stratigraphique) } \\
\text { / non }\end{array}$ & $\begin{array}{l}\text { surface du } \\
\text { prélèvement }\end{array}$ & qq $10 \mu m^{2}$ & - & non \\
\hline $\begin{array}{c}\text { Microscopie } \\
\text { électronique en } \\
\text { transmission (MET) }\end{array}$ & $\begin{array}{c}\text { Examen } \\
\text { nanoscopique }\end{array}$ & oui $\left(1 \mathrm{~mm}^{3}\right)$ & $\begin{array}{l}\text { zone représentant } \\
\text { le prélèvement }\end{array}$ & qq $10 \AA^{2}$ & - & non \\
\hline $\begin{array}{l}\text { Diffraction des RX } \\
\text { (DRX) }\end{array}$ & $\begin{array}{l}\text { Analyse } \\
\text { structurale }\end{array}$ & $\begin{array}{c}\text { non (surface } \\
\text { plane) / oui } \\
\text { (poudre, coupes } \\
\text { stratigraphiques) }\end{array}$ & surface & $\begin{array}{l}\text { qq } 10 \mu \mathrm{m}^{2} / \mu \mathrm{m}^{2} \\
\text { (avec source } \\
\text { synchrotron) }\end{array}$ & $\begin{array}{c}0,1 \% \text { (avec source } \\
\text { synchrotron) - } \\
100 \%\end{array}$ & oui \\
\hline $\begin{array}{l}\text { Diffraction des } \\
\text { électrons }\end{array}$ & $\begin{array}{l}\text { Analyse } \\
\text { structurale }\end{array}$ & oui $\left(1 \mathrm{~mm}^{3}\right)$ & $\begin{array}{l}\text { zone représentant } \\
\text { le prélèvement }\end{array}$ & qq $10 \AA^{2}$ & $1-100 \%$ & non \\
\hline $\begin{array}{l}\text { Spectrométrie } \\
\text { Raman }\end{array}$ & $\begin{array}{l}\text { Analyse } \\
\text { structurale et } \\
\text { moléculaire }\end{array}$ & non & $\begin{array}{l}\text { surface (sauf en } \\
\text { mode confocal) }\end{array}$ & $\begin{array}{l}\text { qq } \mu m^{2} \text { (possibilité } \\
\text { de balayage ligne et } \\
\text { de cartographie) }\end{array}$ & $5-100 \%$ & oui \\
\hline $\begin{array}{l}\text { Spectrométrie } \\
\text { infrarouge }\end{array}$ & $\begin{array}{l}\text { Analyse } \\
\text { structurale et } \\
\text { moléculaire }\end{array}$ & $\begin{array}{c}\text { oui (1-2 mg) / } \\
\text { non (en réflexion) }\end{array}$ & $\begin{array}{l}\text { zone représentant } \\
\text { le prélèvement }\end{array}$ & $\begin{array}{c}\text { qq } 10 \mu \mathrm{m}^{2} / \mu^{2} \\
\text { (avec une source } \\
\text { synchrotron) }\end{array}$ & $5-100 \%$ & oui \\
\hline $\begin{array}{c}\text { Fluorescence X } \\
\text { (XRF) }\end{array}$ & $\begin{array}{l}\text { Analyse } \\
\text { élémentaire }\end{array}$ & non (sous atm.) & $\begin{array}{l}\text { surface (sauf en } \\
\text { mode confocal) }\end{array}$ & $\begin{array}{l}\text { qq } 10 \mu \mathrm{m}^{2} \text { dans l'air } \\
\text { sub- } \mu \mathrm{m}^{2} \text { sous vide, } \\
\text { cartographies 2D et } \\
\text { 3D possibles }\end{array}$ & $\begin{array}{l}\text { qq } 10 \mathrm{ppm}-100 \% \\
\text { éléments majeurs, } \\
\text { mineurs et traces }\end{array}$ & oui \\
\hline $\begin{array}{l}\text { Proton-induced X- } \\
\text { ray emission (PIXE) }\end{array}$ & $\begin{array}{l}\text { Analyse } \\
\text { élémentaire }\end{array}$ & non (sous atm.) & surface & $\begin{array}{l}\text { qq } \mu \mathrm{m}^{2} \text { dans l'air } \\
\text { sub- } \mu \mathrm{m} \text { sous vide, } \\
\text { cartographies } 2 \mathrm{D} \\
\text { possibles }\end{array}$ & $\begin{array}{l}\text { qq } 10 \mathrm{ppm}-100 \% \\
\text { éléments majeurs, } \\
\text { mineurs et trace }\end{array}$ & non \\
\hline $\begin{array}{l}\text { Analyse par } \\
\text { réaction nucléaire } \\
\text { (NRA) }\end{array}$ & $\begin{array}{l}\text { Analyse } \\
\text { élémentaire et } \\
\text { isotopique }\end{array}$ & non & surface & qq $\mu \mathrm{m}^{2}$ dans l'air & $\begin{array}{l}\text { qq } 10 \text { ppm- } 100 \%, \\
\text { éléments majeurs } \\
\text { et mineurs }\end{array}$ & non \\
\hline $\begin{array}{c}\text { Rétrodiffusion } \\
\text { Rutherford (RBS) }\end{array}$ & $\begin{array}{l}\text { Analyse } \\
\text { élémentaire }\end{array}$ & non & $\begin{array}{l}\text { surface, profil en } \\
\text { profondeur }(200 \AA \\
-1 \mu \mathrm{m})\end{array}$ & qq $\mu \mathrm{m}^{2}$ dans l'air & 1000 ppm & non \\
\hline $\begin{array}{c}\text { Spectroscopie UV- } \\
\text { Visible-IR }\end{array}$ & $\begin{array}{l}\text { Analyse } \\
\text { structurale }\end{array}$ & $\begin{array}{c}\text { oui (en } \\
\text { transmission) } \\
\text { non (en réflexion) }\end{array}$ & $\begin{array}{c}\text { surface (en } \\
\text { réflexion) / globale } \\
\text { (en transmission) }\end{array}$ & $\mathrm{qq} \mathrm{mm}^{2}$ & $10-100 \%$ & oui \\
\hline $\begin{array}{c}\text { Spectroscopie } \\
\text { d'absorption X } \\
\text { (XANES/EXAFS) }\end{array}$ & $\begin{array}{c}\text { Analyse } \\
\text { structurale }\end{array}$ & non / oui (qq mg) & $\begin{array}{l}\text { surface (en mode } \\
\text { fluorescence) } \\
\text { globale (en } \\
\text { transmission) }\end{array}$ & $\mathrm{qq} \mu \mathrm{m}^{2}$ & traces $-100 \%$ & non \\
\hline $\begin{array}{l}\text { Spectroscopie } \\
\text { Mössbauer }\end{array}$ & $\begin{array}{l}\text { Analyse } \\
\text { structurale }\end{array}$ & oui $(20-100 \mathrm{mg})$ & globale & $\mathrm{qq} \mathrm{mm}^{2}$ & traces - $100 \%$ & non \\
\hline $\begin{array}{l}\text { Spectroscopie de } \\
\text { luminescence }\end{array}$ & $\begin{array}{c}\text { Analyse } \\
\text { structurale au } \\
\text { niveau } \\
\text { électronique }\end{array}$ & non & surface & qq $\mathrm{mm}^{2}$ & ultratraces - $100 \%$ & non \\
\hline
\end{tabular}

Tableau 1. Aperçu des méthodes d'examen microscopique et d'analyse les plus couramment utilisées pour l'étude des matériaux du patrimoine culturel. 


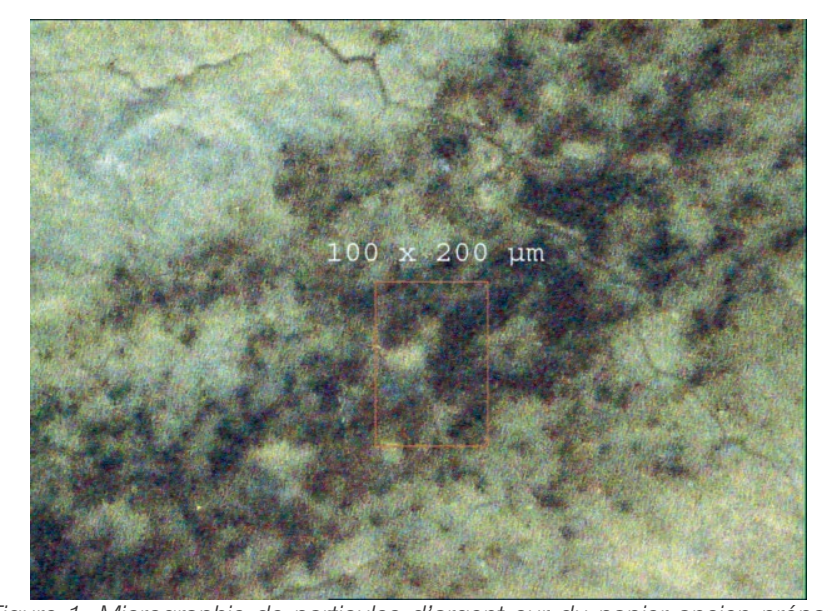

Figure 1. Micrographie de particules d'argent sur du papier ancien préparé (blanc d'os et blanc de Pb), avec la zone d'analyse indiquée.
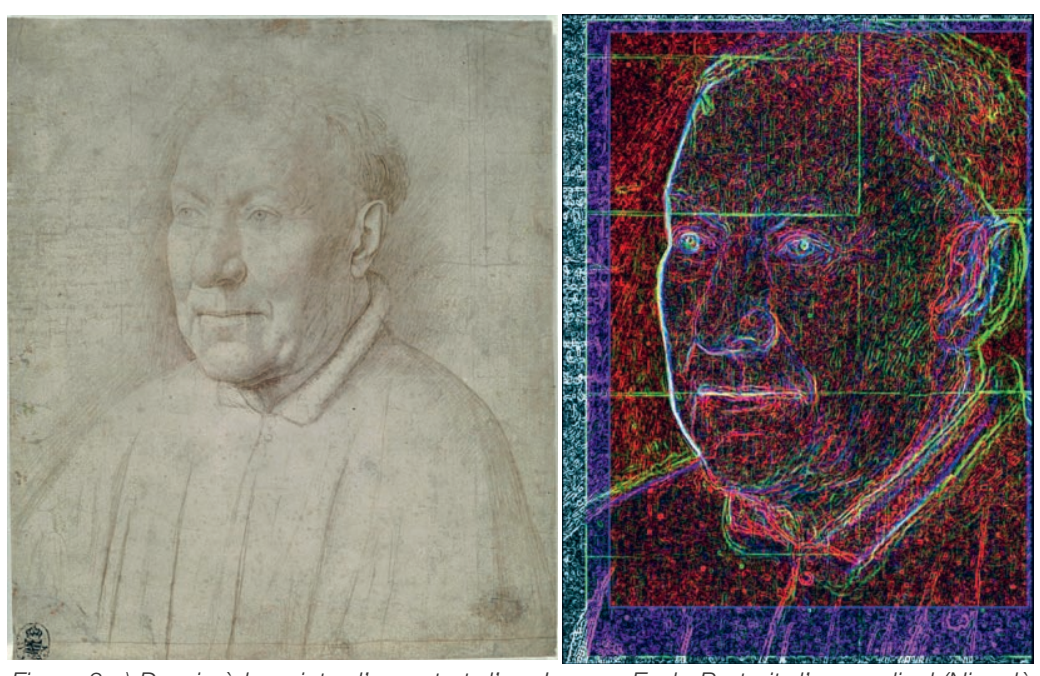

Figure 2 a) Dessin à la pointe d'argent et d'or. Jan van Eyck. Portrait d'un cardinal (Niccolo Albergati ?), $21,2 \times 18 \mathrm{~cm}$, daté d'environ 1435. Cabinet de dessin, Collections nationales de Dresde ( $n^{\circ}$ d'inventaire C775). b) Comparaison de la composition du dessin préparatoire et du tableau final : superposition du dessin agrandi par un facteur 1,4 (en rouge) et du tableau correspondant (en bleu). Le tableau est conservé au Kunsthistorisches Museum de Vienne.

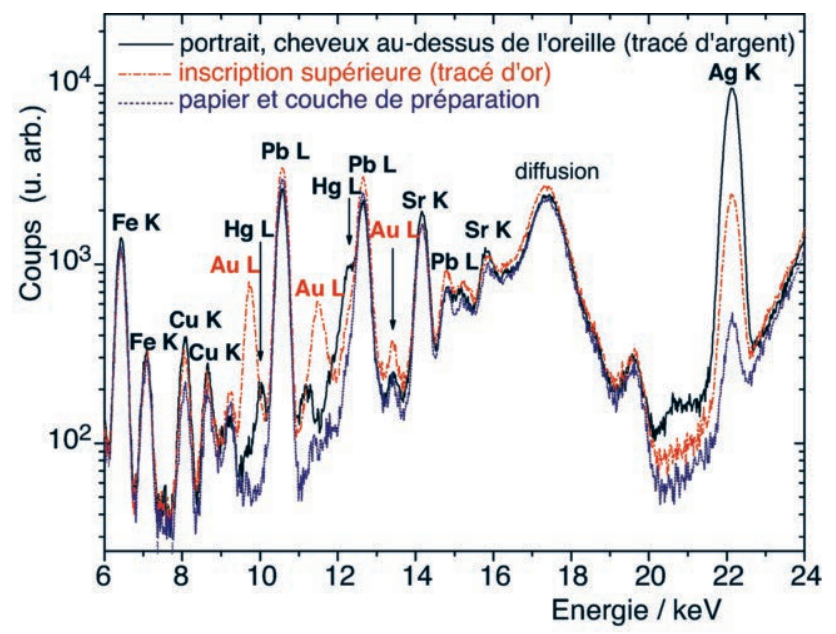

Figure 3. Spectres de fluorescence $X$ induite par rayonnement synchrotron, mesurés sur le dessin à la pointe de métal de Jan van Eyck (figure 2a). Courbe en pointillés bleus : analyse du papier et de la couche de préparation à base de blanc d'os (hydroxylapatite, un phosphate de Ca) et de traces de blanc de $\mathrm{Pb}$; on y observe les éléments $\mathrm{Fe}, \mathrm{Cu}$, Sr et Pb. Courbe en tirets rouges : analyse d'une annotation à la pointe d'or (difficile à voir, en haut de la figure 2a). Courbe en trait continu noir : analyse des cheveux au-dessus de l'oreille du portrait ; on y détecte principalement les éléments Ag et $\mathrm{Hg}$. te par rayonnement synchrotron (SY-FX). Nous l'avons mise en œuvre sur la BAMline de la source synchrotron BESSY de Berlin. L'œuvre est placée devant la sortie du faisceau de rayons $\mathrm{X}$, dont l'énergie est (généralement) de $33 \mathrm{keV}$ et dont le diamètre est d'une centaine de micromètres. Une table motorisée permet de choisir l'endroit à analyser sur le dessin. Un détecteur assure la collection des rayons $X$ caractéristiques de chaque élément présent et délivre rapidement la composition chimique des matériaux dans le volume étudié. Ce dispositif renseigne donc sur la composition élémentaire des tracés, sans qu'il y ait ni contact avec l'instrument d'analyse, ni dommage pour l'œuvre. Même si la SY-FX n'est pas une méthode d'analyse de surface, le flux très intense du rayonnement incident permet d'obtenir une réponse suffisante du matériau des tracés [10]. Cette technique SY-FX a été, entre autres, employée pour l'analyse d'un dessin très connu.

Le Portrait d'un homme âgé, conservé au Cabinet des gravures de Dresde, en Allemagne, est un dessin très important, car il a été réalisé avec une maîtrise exceptionnelle et, même s'il n'est pas signé, est le seul de cette époque dont on connaisse l'auteur avec certitude : le Flamand Jan Van Eyck (1390-1441). En effet, il s'agit d'un dessin préparatoire pour un tableau du maître, conservé au Musée d'histoire de l'art de Vienne. De nombreuses questions concernent la genèse de l'œuvre et la transposition du dessin sur le tableau (figure 2a-b).

Les analyses par SY-FX ont révélé l'emploi de trois stylets différents : deux d'entre eux sont des stylets d'argent, le deuxième contenant aussi $25 \%$ de cuivre, et le troisième est un stylet d'or, contenant $20 \%$ d'argent (figure 3) [12]. II est à noter que parmi les 100 dessins analysés dans le cadre de ce programme de recherche, deux seulement ont été exécutés avec plusieurs stylets. En outre, Van Eyck est le seul à avoir employé un stylet d'or. Si l'emploi de ce métal précieux était mentionné dans la littérature de l'époque, il n'avait encore jamais été mis en évidence dans un dessin. Il a sans doute été choisi pour ses propriétés mécaniques : l'or, offrant un contact plus doux que l'argent avec le papier, est plus facile à utiliser lorsque l'on recherche la précision, ainsi que pour écrire. De plus, les tracés à la pointe d'or sont gris, tandis que ceux à la pointe d'argent deviennent rapidement bruns. Ainsi, Van Eyck semble avoir exploité différents stylets pour réaliser différents effets.

Les analyses ont permis de retrouver comment Van Eyck a pu procéder : il a pris une feuille de papier déjà préparée, puis un stylet d'argent très pur. II a dessiné les contours du visage, les traits et les cheveux, puis la robe et la limite basse du portrait sous la forme d'un trait horizontal. Prenant un autre stylet d'argent plus riche en cuivre, il a créé une impression de relief en réalisant les ombres des deux côtés du visage. Enfin, il a écrit quelques anotations avec un stylet d'or, concernant la carnation de son sujet, la couleur des yeux, etc. Avec cette même pointe d'or, il a renforcé certains traits du visage, comme la bouche et la pupille.

À l'occasion de ces analyses, les observations par microscopie optique ont également permis de découvrir comment le dessin avait servi à préparer le tableau. L'agrandissement des tracés du dessin se superpose presque exactement au dessin sous-jacent du tableau mis en évidence par réflectométrie infrarouge, ainsi qu'au tableau (figure 2b). Certains spécialistes penchaient en faveur d'un système de transfert optique (avec une lentille), tandis que d'autres évoquaient l'emploi d'une grille ou d'un pantographe (un instrument constitué de quatre tiges articulées, permettant de reproduire mécaniquement un dessin, éventuellement en l'agrandissant ou en le réduisant). Mais l'analyse au microscope a révélé de petits trous en bordure de l'œil gauche, aux coins de la 
bouche, sur le lobe de l'oreille... II est donc possible que Van Eyck ait utilisé un compas pour agrandir le dessin préparatoire. Cette conclusion est confortée lorsque l'on considère l'échelle du portrait par rapport au dessin : 1,4. Cette valeur correspond à la racine carrée de 2 . Une fois le compas ouvert à la longueur à agrandir, il suffisait de la reporter dans deux directions perpendiculaires. La diagonale du carré ainsi formé donnait alors la longueur agrandie du tableau. Ainsi, grâce à cet ensemble d'observations scientifiques, la méthode de travail dans l'atelier de Jan Van Eyck a pu être dévoilée [13].

\section{Étude de la technique picturale indienne grâce à la micro-FX-3D}

L'Inde a une longue tradition de peinture de miniatures. Cette technique artistique était à son apogée lors de la période Moghol. Des scènes de genre ou des souverains Moghol sont représentés sur ces peintures, qui ont été fabriquées dans des ateliers particuliers dans le Nord de I'Inde, du XVI ${ }^{\mathrm{e}}$ au XIXe siècle. Peu de connaissances étaient disponibles jusqu'à présent sur la composition de la palette picturale et la technique de peinture. Dans le but de caractériser ces miniatures, une méthodologie analytique a été mise en place, consistant à combiner différentes méthodes d'examen et d'analyse non-destructives complémentaires. L'émission $X$ induite par particules chargées (PIXE) en mode de faisceau extrait permet l'analyse élémentaire des pigments inorganiques [6]. L'observation sous lumière UV et la spectroscopie d'absorption optique en lumière visible (VIS) permettent celle des colorants organiques. De plus, quelques miniatures ont été analysées en fonction de la profondeur, sans prélèvement, par un nouveau dispositif expérimental, la microfluorescence $X$ résolue en trois dimensions (« micro-FX-3D », voir encadré 1).

Une trentaine de miniatures du style Moghol, datant du XVI ${ }^{\mathrm{e}}$ au XIX siècle, ont été ainsi analysées. Parallèlement, il a été nécessaire d'analyser également des pigments de référence appliqués sur du papier coton. Quelques pigments comme le lapis n'ont pas pu être déterminés de façon sûre par l'analyse élémentaire. Dans ce cas, l'observation sous lumière UV ou la spectroscopie VIS ont permis de les identifier grâce à une fluorescence ou à un spectre optique caractéristiques (figure 5). De même, quelques matériaux n'ont pas pu être déterminés par ces techniques classiques, à cause de la superposition de différentes couches picturales. Dans ce cas, la micro-FX-3D a été utilisée. Par l'analyse des pentes de profils, cette technique a de plus permis de déterminer l'épaisseur des couches picturales et donc d'obtenir des informations précieuses sur l'organisation de ces couches (figure 6).

Ces analyses ont montré que la palette picturale de ces miniatures est composée d'azurite, de bleu de Prusse, d'indigo et de lapis (outremer) pour les bleus, de pigments à base

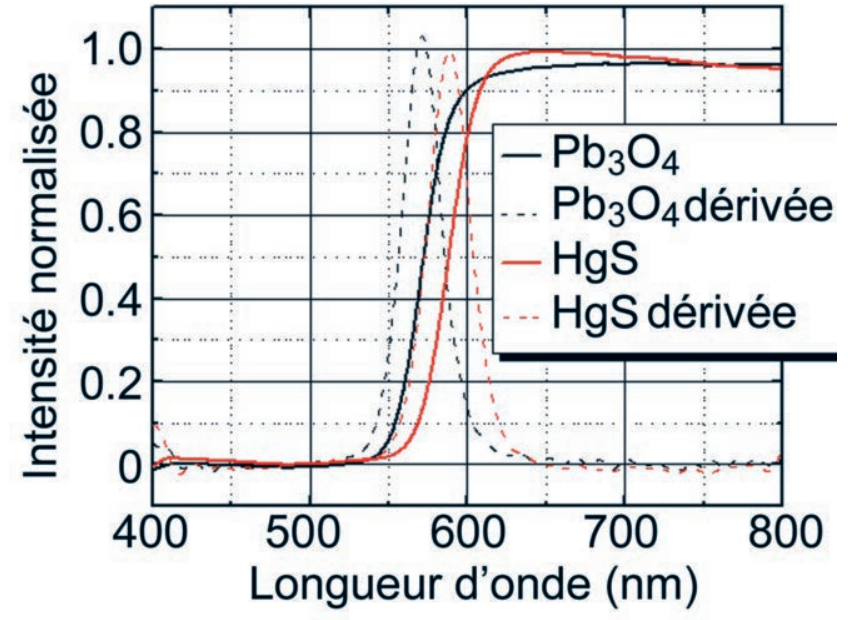

Figure 5. Comparaison des spectres d'absorption optique de deux pigments rouges (minium $\mathrm{Pb}_{3} \mathrm{O}_{4}$, et cinabre, $\mathrm{HgS}$ ). Courbes continues : intensités réfléchies en fonction de la longueur d'onde. Courbes en tirets : dérivées des courbes continues. (C) O.Hahn, BAM Berlin

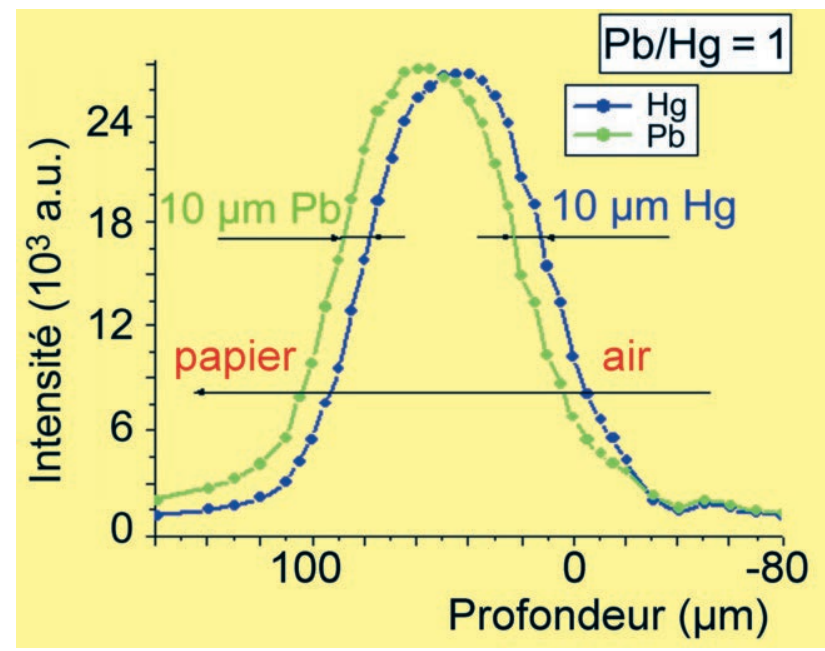

Figure 6. Profil élémentaire en profondeur mesuré directement sur une miniature ( $n^{\circ}$ d'inventaire MIK I 5004(3)) montrant deux couches picturales successives (du cinabre, HgS, sur du blanc de plomb, $\mathrm{Pb}\left(\mathrm{CO}_{3}\right)_{2} \cdot \mathrm{Pb}(\mathrm{OH})_{2}$ ). Le recouvrement des deux profils des éléments $\mathrm{Hg}$ et $\mathrm{Pb}$ est lié à la limite de la résolution spatiale en profondeur (20-30 $\mu \mathrm{m})$. L'épaisseur des couches successives peut être évaluée à $10 \mu \mathrm{m}$ chacune.

\section{Encadré 1}

\section{La microfluorescence $X 3 D$}

Ce dispositif, récemment mis au point, qui permet une analyse élémentaire résolue en profondeur, est basé sur un système d'analyse confocal utilisant deux optiques focalisantes, l'une dans le canal d'excitation et l'autre dans le canal de détection des rayons $X$. Grâce à la superposition des foyers de deux lentilles focalisantes, il est possible de définir un microvolume d'analyse avec une résolution spatiale d'une dizaine de $\mu \mathrm{m}$ (figure 4). En déplaçant l'échantillon, une analyse de surface ou en profondeur devient possible. La limite de la profondeur d'analyse dépend de la source de RX excitatrice et de la composition chimique de la matrice de l'échantillon ; elle se situe généralement autour de quelques centaines de $\mu \mathrm{m}$.

Les premières expériences de micro-FX 3D ont été effectuées sur des couches de peintures indiennes. Elles ont montré que ce dispositif était parfaitement adapté à l'analyse non-destructive des matériaux du patrimoine [13] et qu'il permettait notamment d'étudier les techniques picturales anciennes.

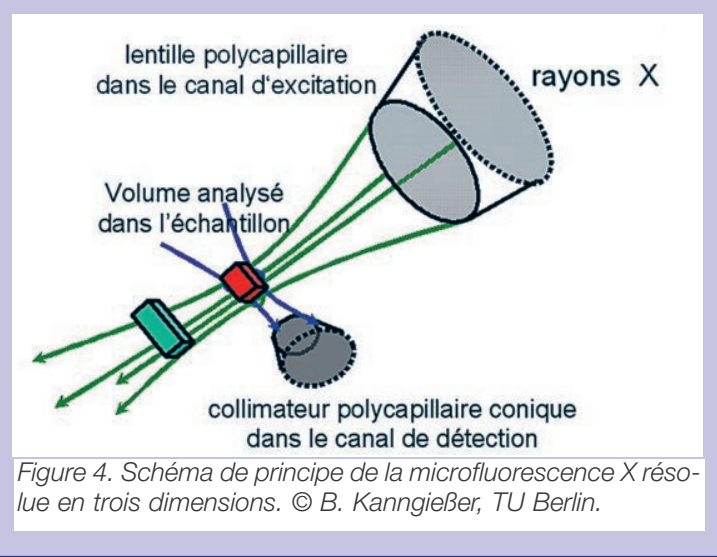


de $\mathrm{Cu}$ (malachite, atacamite), de terres vertes, de mélanges d'indigo et de jaune indien pour le vert, d'ocre et de jaune indien pour le jaune, de minium pour l'orange, de cinabre, d'ocre et de minium, de carmine, de kermesse, de laque et de garance pour le rouge, d'un mélange de carmine et d'indigo ou de kermesse et d'indigo pour le violet ainsi que du blanc de plomb, d'étain et de zinc pour les blancs. Les pigments noirs, probablement à base de produits organiques, n'ont pas pu être identifiés. De plus, les miniatures contiennent de l'argent et de l'or.

L'utilisation de pigments à base d'étain et de zinc a donc été mise en évidence dans différentes couches picturales des miniatures. On pourrait alors croire qu'il s'agissait de faux car, selon nos connaissances, ces pigments n'avaient pas été détectés sur des miniatures indiennes de cette époque. Cependant, cette découverte ne permet pas de conclure directement à la présence d'une copie parmi les miniatures analysées, car d'une part le blanc d'étain a été utilisé dans

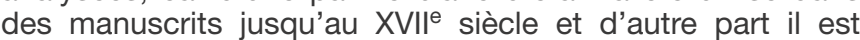
connu par des études archéo-métallurgiques que des minerais de zinc ont déjà été extraits des mines du Nord-Ouest de I'Inde vers 500 avant J.-C. [15-16].

Ces résultats ont permis de mieux caractériser la technique picturale des miniatures indiennes et illustrent l'importance de croiser différentes méthodes d'analyse complémentaires pour l'étude des objets du patrimoine ; elles montrent plus particulièrement les perspectives ouvertes en archéométrie par la mise au point d'une nouvelle méthode analytique : la microfluorescence $X$ résolue dans les trois dimensions.

\section{Étude des phénomènes d'altération, des procédés de fabrication des matériaux et de leur couleur}

\section{Des défenses de mastodonte fossiles à la turquoise osseuse}

La turquoise osseuse, aussi nommée odontolite, fut utilisée comme pierre semi-précieuse pour orner des objets d'art au Moyen-Âge. Elle n'a pas été reconnue tout de suite comme différente de la turquoise minérale, car la couleur et l'aspect de ces deux gemmes sont très similaires. Au XVII e siècle, il a été remarqué qu'il pouvait s'agir d'os ou d'ivoire fossiles chauffés, matériaux généralement composés de phosphates de calcium (hydroxyl- ou fluorapatite). Par la suite, de nombreuses hypothèses sur l'origine de la couleur de la turquoise osseuse ont été émises par des naturalistes réputés du XVIII siècle, comme Réaumur [17] ou par des gemmologistes contemporains.

Récemment, nous avons développé une méthodologie analytique, fondée sur une combinaison d'analyses physico-chimiques et spectroscopiques, pour élucider la nature exacte de ce matériau et l'origine de sa couleur. Les analyses ont été appliquées à des objets provenant des collections médiévales du Musée du Louvre et du Musée national du Moyen-Âge (Musée de Cluny), ainsi qu'à des «pierres » brutes du Muséum national d'Histoire naturelle.

La composition chimique a été étudiée au niveau des éléments majeurs, mineurs et en traces par microscopie électronique à balayage couplée à un système d'analyse $X$ dispersif en énergie (MEB-EDX) et par micro-PIXE/PIGE (émission gamma induite par particules chargées) à l'accélérateur de particules AGLAE [6]. Ces analyses ont permis de distinguer deux types de turquoises osseuses : d'une part de l'os (de l'apatite) coloré par des sels de cuivre (" fausse turquoise osseuse »), d'autre part un matériau osseux riche en fluor, sans traces de cuivre, mais contenant du fer, du manganèse et quelques autres éléments à l'état de traces (« vraie turquoise osseuse »). Ces deux matériaux se différencient également par leur cristallinité : la fausse turquoise osseuse présente une très faible cristallinité, proche de celle de l'os moderne, contrairement à la vraie turquoise osseuse, relativement bien cristallisée. La morphologie et la taille des cristaux d'apatite des échantillons de vraie turquoise osseuse et des vestiges osseux chauffés à $500-700{ }^{\circ} \mathrm{C}$, révélées par microscopie électronique en transmission (MET), sont similaires. La vraie turquoise osseuse correspond donc bien à des échantillons d'os ou d'ivoire chauffés à environ $600^{\circ} \mathrm{C}$. Cependant, ces données, qui permettent de mieux définir la nature de la turquoise osseuse, n'apportent aucun élément de réponse sur l'origine de sa couleur.

D'autres méthodes spectroscopiques étaient donc nécessaires pour élucider ce phénomène. La luminescence induite par laser (TRLIF), la spectrométrie UV-Vis-NIR et la spectroscopie d'absorption X (XANES et EXAFS, voir encadré 2) ont montré que cette couleur turquoise est liée à la présence de traces d'ions $\mathrm{Mn}^{5+}$ dans un environnement tétraédrique oxygéné [18] (figure 7).

L'évolution de la couleur a aussi été suivie en fonction de la température de chauffage : l'apatite recristallise au cours du chauffage et les ions $\mathrm{Mn}^{2+}$ se transforment en ions $\mathrm{Mn}^{5+}$. Cette oxydation est accompagnée d'un changement structural : les ions $\mathrm{Mn}^{2+}$ dans un site proche d'une symétrie octaédrique passent à du $\mathrm{Mn}^{5+}$ dans un site tétraédrique régulier. La formation des ions $\mathrm{Mn}^{5+}$, dans un site équivalent à celui du $\mathrm{P}^{5+}$ dans la maille apatitique, est responsable de l'apparition de la couleur bleue turquoise de ce matériau [19]. Ce phénomène peut apparaître dans d'autres matériaux apatitiques, tels que des apatites géologiques, et n'est donc pas restreint à l'apatite biogénique.

Ainsi, la formation de la turquoise osseuse a pu être reconstituée. Une première étape de fossilisation naturelle du matériau osseux (os, ivoire, dent), qui inclut l'enrichissement en certains éléments traces comme le fluor, le fer et le manganèse, est suivie d'un chauffage à environ $600^{\circ} \mathrm{C}$ sous atmo-

\section{Encadré 2}

\section{Spectroscopie d'absorption de rayons $X$ (XANES/EXAFS)}

Le seuil d'absorption de rayons $X$ d'un élément chimique dans un solide (par exemple seuil $K$ correspondant à l'éjection d'un électron $1 \mathrm{~s}$ vers des états inoccupés) est sensible à son état d'oxydation : pour un cation, une augmentation de l'état de valence se traduit par un déplacement du seuil vers les hautes énergies.

La forme du coefficient d'absorption autour du seuil, appelée XANES (X-ray absorption near-edge structure). Dans les oxydes, une structure appelée « prépic " peut être observées juste en dessous du seuil. Au seuil K des ions de métaux de transition $3 d$ faiblement concentrés, ces prépics sont dus à des transitions quadrupolaires $1 s \rightarrow 3 d$ et/ou à des transitions dipolaires vers des états hybridés avec les états $3 d$ de l'atome absorbeur (voir figure 7).

Pour des énergies de photon plus grandes (entre 50 et $1000 \mathrm{eV}$ typiquement), des oscillations du coefficient d'absorption, appelées EXAFS (Extended X-ray absorption fine structure) sont, observées. L'EXAFS correspond à la fonction d'onde du photoélectron qui est la somme d'une partie émise et d'une partie rétrodiffusée par les atomes voisins : son analyse renseigne sur l'environnement (coordinance, distances) de l'atome central. 


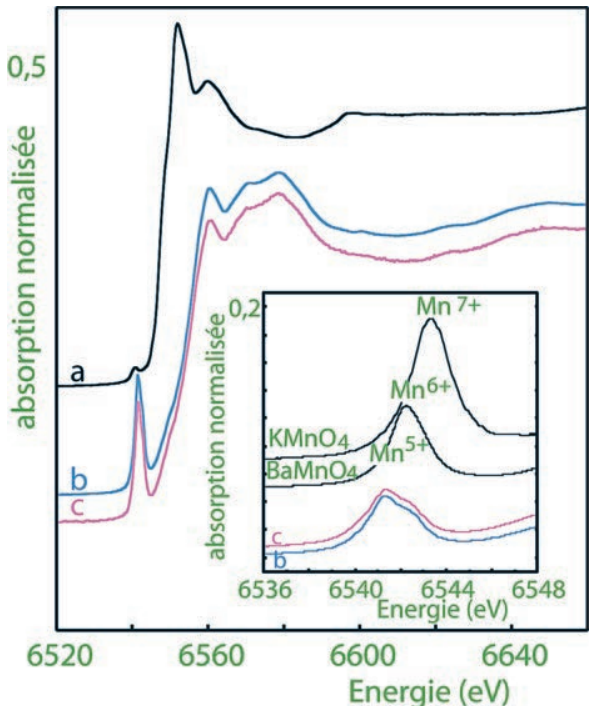

Figure 7.

Comparaison de spectres XANES au seuil K du Mn : a) de l'ivoire fossile, b) de l'ivoire fossile chauffé à $600^{\circ} \mathrm{C}$, et c) d'une turquoise osseuse. Le coefficient d'absorption $X$ du matériau est reporté en fonction de l'énergie des photons incidents. Dans l'encart, les "prépics 》 des différents spectres sont représentés en ordre inverse pour une meilleure lisibilité de leur décalage en énergie en fonction de l'état de valence du Mn.

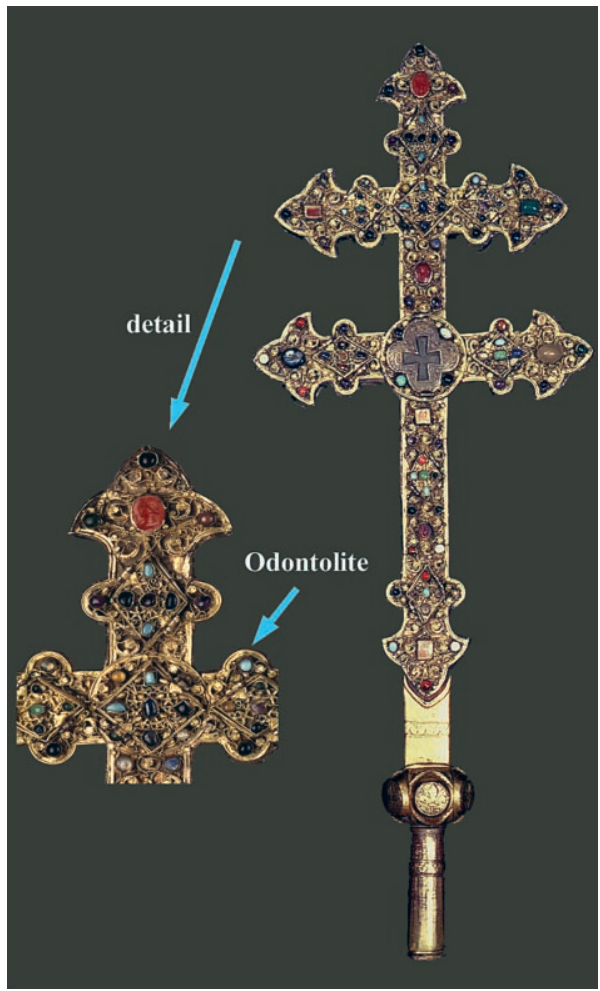

Figure 8

Croix reliquaire de la Vraie Croix contenant une turquoise osseuse. XIII siècle. Musée National du Moyen Âge. (C) C2RMF

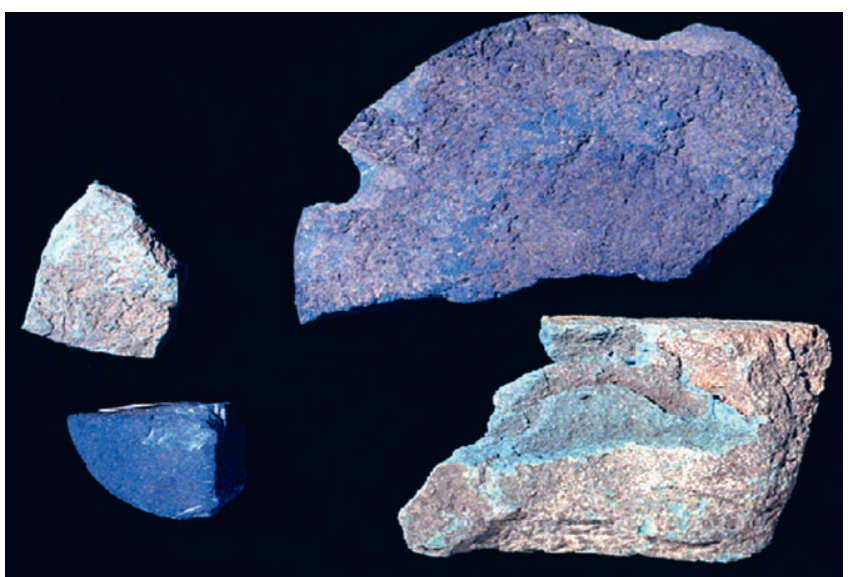

Figure 9. Échantillons de vert et de bleu égyptiens archéologiques. Pains bruts provenant de Deir el Medineh, Egypte (XVIIle dynastie).

(C) C2RMF, D. Vigears. sphère oxydante formant des ions $\mathrm{Mn}^{5+}$, responsables de la couleur turquoise. Puis le matériau coloré a été taillé et appliqué comme décoration avec d'autres gemmes sur les objets d'art (figure 8).

\section{Vert et bleu égyptiens : deux pigments synthétiques très anciens}

Un pigment bleu contenant du calcium, du cuivre et du silicium, dont on ne connaît pas d'équivalent naturel, a été identifié sur des monuments égyptiens. Ce «bleu égyptien » a été créé durant l'Ancien Empire en Egypte vers 2600 avant J. C. comme imitation du lapis-lazuli, et utilisé jusqu'au VII ${ }^{\mathrm{e}}$ siècle après J. C. dans toute la Méditerranée et même dans le Nord de l'Europe. Depuis, de nombreuses investigations ont été consacrées à sa caractérisation, à son procédé de fabrication et à l'origine de sa couleur. Malheureusement, aucune représentation ou trace écrite de l'époque égyptienne, relative à la technologie de fabrication de ce pigment, n’a été conservée jusqu'à nos jours.

En parallèle au bleu égyptien, un autre pigment de couleur turquoise, avec une composition chimique très similaire, le " vert égyptien ", était apparu sur des décors en Egypte un peu plus tardivement. À cause de la similarité des deux pigments, ce vert égyptien n'a pas été reconnu immédiatement comme un nouveau pigment synthétique, mais a été souvent considéré comme un produit d'altération du bleu égyptien.

Grâce à des méthodes d'analyses complémentaires (MEB/MET-EDX, diffraction des rayons X, spectroscopies micro-Raman et UV-Visible, voir tableau 1), les deux pigments ont pu être différenciés sans ambiguïté [20]. Les échantillons archéologiques étudiés étaient conservés au Département des Antiquités égyptiennes du musée du Louvre ; il s'agissait de matériaux bruts provenant d'un village d'artisans, Deir el Medineh, datant de la XVIII ${ }^{\mathrm{e}}$ dynastie du Nouvel Empire (figure 9). Ces pigments étaient destinés à la décoration des monuments de la Vallée des Rois. Les analyses ont montré que le bleu et le vert égyptiens sont des matériaux composites, constitués d'une fraction cristalline et d'une fraction amorphe ; ils se différencient par la nature des phases cristallines (quartz, tridymite, cuprorivaïte pour le bleu, quartz, cristobalite, parawollastonite pour le vert) et par la composition de la phase amorphe (riche en cuivre dans le cas du vert).

Grâce à la reproduction des pigments en laboratoire, il a été possible de reconstituer les conditions des procédés de fabrication et de fournir une preuve irréfutable que le vert égyptien diffère du bleu égyptien. Les mélanges nécessaires à l'obtention du vert égyptien montrent qu'il faut des proportions rigoureuses, avec une quantité de fondant $\left(\mathrm{NaCO}_{3}\right)$ supérieure à celle du bleu égyptien. De plus, le vert égyptien se forme à plus haute température que le bleu égyptien, ce qui permet clairement d'expliquer pourquoi le vert ne peut pas résulter d'une altération (processus qui se déroule généralement à basse température) du bleu.

Même si de nombreuses recherches avaient déjà été réalisées sur ces pigments, on disposait de peu de données précises sur l'origine de leur couleur. Selon les hypothèses avancées jusqu'alors, les ions $\mathrm{Cu}^{2}$, présents dans la phase cuprorivaïte $\left(\mathrm{CuCaSi}_{4} \mathrm{O}_{10}\right)$, étaient responsables de la couleur bleue, tandis que la couleur du vert égyptien était expliquée par la présence d'une phase amorphe riche en cuivre et en fer

Une méthode particulièrement bien adaptée à l'analyse fine de l'état de valence et de l'environnement atomique d'ions de métaux de transition, est la spectroscopie d'absorption X (voir tableau 1 et encadré 2). Des expériences de XANES et d'EXAFS ont donc été réalisées au seuil $K$ du cuivre d'échantillons de bleu et de vert égyptiens archéologiques, sur la ligne KMC2 du synchrotron BESSY (Berlin, Allemagne). La position en énergie du seuil, située vers 8986,7 eV dans les deux pigments, correspond à celle des ions $\mathrm{Cu}^{2+}$. Le spectre du bleu 
montre plus d'oscillations, ce qui suggère que les ions cuivre appartiennent à une phase cristalline. L'analyse des données d'EXAFS permet de déterminer avec précision l'environnement des ions de cuivre dans le bleu égyptien, le meilleur ajustement du spectre expérimental avec un spectre calculé ayant été obtenu avec le modèle structural bien connu de la cuprorivaïte. Pour le vert égyptien, où le site du cuivre n'était pas bien connu, un bon ajustement du spectre a montré que les ions $\mathrm{Cu}^{2+}$ se trouvent dans un environnement octaédrique distordu (effet Jahn-Teller). En conclusion, c'est l'environnement chimique différent du cuivre dans le bleu et le vert égyptiens, et non pas la présence supplémentaire d'un autre chromophore dans le vert égyptien, qui est responsable des nuances de couleur des deux pigments [21].

\section{Conclusion}

Les études physico-chimiques du patrimoine culturel représentent un véritable défi analytique. Dans de nombreux cas, il est nécessaire d'avoir recours à des méthodes de microanalyse, parfois même en utilisant de "grands instruments" comme les accélérateurs de particules ou le rayonnement synchrotron. En effet, les empreintes caractéristiques de l'origine, du procédé de fabrication ou du processus d'altération d'un matériau, sont souvent les phases mineures, micro- ou nanoscopiques, ou les éléments présents localement ou en très faibles concentrations. Notons qu'une tendance actuelle porte sur le développement de systèmes de microfluorescence ou/et de diffraction $\mathrm{X}$ portables, pour des recherches in situ sur les sites ou dans les musées.

Enfin, il faut rappeler que ces recherches en archéométrie ne peuvent pas être menées sans une étroite collaboration entre chercheurs de différents horizons, car l'interprétation des résultats analytiques nécessite de prendre en compte l'ensemble des données disponibles sur les objets, qu'elles soient historiques, archéologiques, physico-chimiques ou biologiques.

\section{Remerciements}

Les travaux de recherche présentés dans cet article ont pu être réalisés grâce à la collaboration avec de nombreuses équipes de recherche françaises et allemandes. Je tiens particulièrement à remercier Silke Merchel et Martin Radtke, BAM Berlin, Birgit Kanngießer et Wolfgang Malzer, TU Berlin, Alain Duval et Sandrine Pagès-Camagna, C2RMF, ainsi que Christian Brouder, Guillaume Morin et Delphine Cabaret, IMPMC Paris. Yves Adda est vivement remercié pour ses conseils lors de la rédaction. Cet article est dédié, pour son départ en retraite, à Colette Vignaud, qui a largement contribué aux recherches par microscopie électronique en transmission appliquée au patrimoine culturel.

\section{Références}

[1] M. Regert, M.-F. Guerra, I. Reiche (2006) « Analyses physico-chimiques des matériaux du patrimoine culturel : Objectifs, principes, méthodes et exemples d'application ", Techniques de l'Ingénieur 3, Partie 1, P3780, 1-35, et Partie 2, P 3781, 1-20.

[2] http://carbon14.univ-lyon1.fr/

[3] R.E. Taylor, M.J. Aitken (1997) Chronometric Dating in Archaeology, Kluwer Academic Publishers, Norwell, USA.

[4] E. Willerslev, A. Cooper (2005) "Review Paper: Ancient DNA", Proc. R. Soc. B $272,3-16$.

[5] J.M. Dupouy (1996) « Les méthodes d'investigation scientifique et le patrimoine artistique » Bulletin de la SFP 107, 23-26.

[6] J.-C. Dran (2002) « Plein feu sur les objets d'art et d'archéologie avec l'accélérateur de particules du Louvre ", Bulletin de la SFP $136,12-17$.

[7] E. Dooryhée (2003) "Advances in art and archaeology using X-ray synchrotron radiation" in Molecular and Structural Archaeology, NATO Science Series, edited by G. Tsoucaris and J. Lipkowski, Kluger Academic Publishers, 201-209.

[8] P. Martinetto et al. (2003) "Synchrotron et matériaux du patrimoine : les cosmétiques de l'Egypte ancienne ", Bulletin de la SFP $138,4-7$.
[9] M. Regert (2004) "Investigating the history of prehistoric glues through gas chromatography - mass spectrometry" Journal of Separation Science 27, 244-254.

[10] I. Reiche, A. Berger, W. Görner, S. Merchel, M. Radtke, J. Riederer, H. Riesemeier, M. Roth (2004) "Following the traces of Albrecht Dürer: Analysis of silver point drawings by spatially resolved synchrotron-induced X-ray fluorescence analysis", Nuclear Instruments and Methods in Physics B 226, 83-91.

[11] A. Duval, H. Guicharnaud, J.C. Dran (2004) "Particle induced Xray emission: a valuable tool for the analysis of metalpoint drawings" Nuclear Instruments and Methods in Physics B 226, 60-74.

[12] I. Reiche, A. Berger, W. Görner, T. Ketelsen, S. Merchel, M. Radtke, J. Riederer, H. Riesemeier, M. Roth (2004) "Spatially resolved Synchrotron-induced X-ray fluorescence analyses of metal point drawings and their mysterious inscriptions", Spectrochimica Acta B 59, 1657-1662.

[13] T. Ketelsen, I. Reiche, O. Simon, S. Merchel (2005) "New information on Jan van Eyck's portrait drawing in Dresden", The Burlington Magazine CXLVII, $\mathrm{n}^{\circ} 1224,170-175$.

[14] B. Kanngießer, W. Malzer, I. Reiche (2003) "A new 3D micro X-ray fluorescence analysis set-up - First archaeometric applications", Nuclear Instruments and Methods in Physics Research B 211, 259-264.

[15] I. Reiche, R. Britzke, G.Bukalis, U. Reinholz, H.-P. Weise, R.D. Gadebusch (2005) "An external PIXE study: Mughal painting pigments", X-Ray Spectrometry 34, 42-45.

[16] P. T. Craddock (1987) "The early history of zinc", Endeavour, New Series 11, 183-191.

[17] M. de Réaumur (1715) «Observations sur les Mines de Turquoises du Royaume; sur la nature de la Matière qu'on y trouve ; et sur la matière dont on lui donne la couleur ", Paris, Académie Royale des Sciences, 174-202.

[18] I. Reiche, C. Vignaud, B. Champagnon, G. Panczer, C. Brouder G. Morin, V.A. Solé, L. Charlet, M. Menu (2001) "From Fossil Mastodon Ivory to Gemstone. The colour origin of odontolite - a mysterious imitation of turquoise", American Mineralogist 86, 1519-1524

[19] I. Reiche, G. Morin, C. Brouder, C. Vignaud, T. Calligaro and M. Menu (2002) "Manganese Accommodation in Fossilised Mastodon Ivory and Heat Induced Colour Transformation: Evidence by EXAFS", European Journal of Mineralogy 14, 10691073.

[20] S. Pagès-Camagna, S. Colinart (2003) "The Egyptian Green Pigment: Its manufacturing process and links to Egyptian Blue", Archaeometry 45, 4, 637-658. 